\title{
Laparoscopic splenectomy and devascularization for massive splenomegaly in portal hypertensive patients: a retrospective study of a single surgical team's experience with 6-year follow-up data
}

\author{
Dong Wang ${ }^{1 \#}$, Xiao Chen ${ }^{1 \#}$, Ling Lv", Tao Yang ${ }^{1}$, Bo Huang ${ }^{1}$, Yanlong Cao ${ }^{1}$, Jianguo Lu ${ }^{1}$, Jikai Yin $^{1 \wedge}$ \\ ${ }^{1}$ Department of General Surgery, Tangdu Hospital, Fourth Military Medical University, Xi'an, China; ${ }^{2}$ Department of Disease Prevention and \\ Health Care, Tangdu Hospital, Fourth Military Medical University, Xi'an, China \\ Contributions: (I) Conception and design: D Wang, J Lu, J Yin; (II) Administrative support: None; (III) Provision of study materials or patients: D \\ Wang, J Yin, T Yang, B Huang, Y Cao; (IV) Collection and assembly of data: X Chen, L Lv; (V) Data analysis and interpretation: X Chen, L Lv; (VI) \\ Manuscript writing: All authors; (VII) Final approval of manuscript: All authors. \\ \#These authors contributed equally to this work. \\ Correspondence to: Jikai Yin; Jianguo Lu. Department of General Surgery, Tangdu Hospital, Fourth Military Medical University, 569 Xin Si Road, \\ Xi'an 710038, China. Email: tdyjk07@fmmu.edu.cn; ljg1963fmmu@163.com.
}

Background: In China, laparoscopic splenectomy and esophagogastric devascularization (LSED) are
effective and safe tools that are used to treat esophageal-fundic variceal bleeding with portal hypertension
(PHT) with minimal trauma; however, due to the increased difficulty of operation, their application in
massive splenomegaly (MS) remains limited. This study sought to determine the efficacy and safety of LSED
in treating MS patients with PHT. Methods: The data of 124 patients who underwent LSED by a single surgical team at our department from January 2015 to December 2020 were retrospectively analyzed. The characteristics of the patients, perioperative parameters, and long-term follow-up data were examined.

Results: A total of $61 \mathrm{MS}$ and 63 mild-to-moderate splenomegaly (MMS) patients underwent LSED. Much larger spleen and significant lower of white blood cells and platelets was found in MS group compared the MMS group preoperation $(\mathrm{P}<0.05)$. The MS group had a significantly longer operation time $(\mathrm{P}=0.009)$, more blood loss $(\mathrm{P}=0.003)$, and more abdominal drainage days $(\mathrm{P}=0.017)$ than the $M M S$ group. Four patients in the MS group and 0 patients in the MMS group were converted to open surgery. No significant difference was found between the 2 groups in terms of postoperative complications. Nine patients in the MMS group and 10 in the MMS group experienced recurrent bleeding in the follow-up period, but no significant differences were observed in terms of recurrent bleeding and overall survival (OS) between the 2 groups.

Conclusions: LSED can be used to treat MS patients with PHT under careful perioperative management. For experienced surgeons, LSED is a safe, feasible, and minimally invasive procedure with satisfactory longterm outcomes that can be used to treat MS patients with PHT.

Keywords: Laparoscopic splenectomy (LS); massive splenomegaly (MS); devascularization; portal hypertension (PHT)

Submitted Dec 07, 2021. Accepted for publication Feb 18, 2022.

doi: $10.21037 / \mathrm{atm}-22-502$

View this article at: https://dx.doi.org/10.21037/atm-22-502

\footnotetext{
$\wedge$ ORCID: 0000-0002-4151-6391.
} 


\section{Introduction}

Portal hypertension (PHT) associated with posthepatic cirrhosis is very common in China. Open splenectomy combined with variceal devascularization has been shown to be an effective and feasible surgical procedure for the treatment of PHT (1-3). With the development of laparoscopic equipment and the laparoscopic technique, several studies have reported that the laparoscopic splenectomy and esophagogastric devascularization (LSED) procedure is more acceptable than, and preferable to, open surgery (4-6). However, the splenomegaly occupies the abdominal space and creates difficulties in the laparoscopic operation. Thus, laparoscopic splenectomy (LS), especially for massive splenomegaly (MS), is a major procedure limiting LSED surgery. Currently, without be updated for more than 10 years, the European Association for Endoscopic Surgery (EAES) guidelines (7) still consider PHT secondary to cirrhosis a contraindication for LS, and recommend that hand-assisted techniques be used to avoid conversion to open surgery, and reduce complication rates in cases of MS. However, with the development of laparoscopic instruments and the experience of surgeons, though, LS remains a technological challenge for MS, spleen size should not be regarded as the only determinant of LS success $(8,9)$. Thus, we present our experience of LSED with an emphasis on safety and feasibility of the laparoscopic surgical procedure for MS in patients with PHT. Additionally, we also analyzed the postoperative esophagogastric re-bleeding rate and overall survival (OS) of patients in the follow-up period. We present the following article in accordance with the STROBE reporting checklist (available at https://atm.amegroups.com/article/ view/10.21037/atm-22-502/rc).

\section{Methods}

\section{Participants and surgical criteria}

The data of 124 patients (71 male and 53 female) diagnosed with cirrhotic PHT who underwent LSED from January 2015 to December 2020 at Department of General Surgery, Tangdu Hospital, Fourth Military Medical University, were retrospectively reviewed. All the baseline patient data are summarized in Table 1. Patients with a long spleen axis $>20 \mathrm{~cm}$ were assigned to the MS group, and those with a long splenic axis $<20 \mathrm{~cm}$ were assigned to the mild-to-moderate splenomegaly (MMS) group. Child-Pugh classification was used for the preoperative assessment of liver function. The hepatic venous pressure gradient (HVPG) was also routinely measured before surgery. To be eligible for the surgery, patients had to meet the following inclusion criteria: (I) have cirrhosis with a history of variceal hemorrhage (hematemesis and melena); (II) have cirrhosis with no history of variceal hemorrhage but have a high risk of variceal hemorrhage [simultaneous grade III esophageal varices, blue varices, or cherry red spots from bleeding varices diagnosed by endoscopy, severe hypersplenism (a white blood cell count $<2.0 \times 10^{9} / \mathrm{L}$ and a platelet count $<50 \times 10^{9} / \mathrm{L}$ )]; (III) have a high HVPG $>12 \mathrm{mmHg}(10,11)$. Patients with severe liver dysfunction (Child-Pugh class C) or hepatocellular carcinoma were excluded, as were patients with incomplete data. All procedures performed in this study involving human participants were in accordance with the Declaration of Helsinki (as revised in 2013). This study was approved by the Ethical Committee of Tangdu Hospital (approval No. 202011-32) and informed consent was taken from all the patients.

\section{Preoperative evaluation and surgical procedure}

Multidetector-row computed tomography was performed to evaluate the portosystemic collateral vessels, spleen size, and locations of the splenic artery and pericardial vessels (see Figure 1A). The spleen volume was measured with Myrian ${ }^{\circledR}$ Studio software (INTRASENSE, Montpellier, France).

Each patient was placed in the supine position with the left flank elevated at a 30-degree angle for the splenectomy. Usually, a 12-mm laparoscopic trocar was first inserted through an incision beneath the umbilicus (to the right or left side according to the spleen size). Four other trocars were inserted under visual control as follows: two $12-\mathrm{mm}$ laparoscopic trocars were separately inserted through the crossover point of the right axillary midline and navel horizontal line, at the level of the lower pole of the spleen; and two 5-mm laparoscopic trocars were inserted at a point along the ventrimeson, $3 \mathrm{~cm}$ below the xiphoid process and through the midpoint between the first location of the 5-mm laparoscopic trocar and the navel. The locations of the trocars were adjusted according to the spleen size. Point 1 was used as the laparoscopic observation hole, Points 2 and 3 as the main operative holes, and Points 4 and 5 as secondary operative holes (see Figure 1B). Electrocautery, the LigaSure ${ }^{\mathrm{TM}}$ vessel-sealing system (Medtronic, Minneapolis, MN, USA), hemostatic clamps, and the Endo GIA $^{\mathrm{TM}}$ stapler (Medtronic) were used for vessel disruption.

The separation and ligation of the splenic artery was the first main procedure in LS (see Figure 1C). Without blood 
Table 1 Patients characteristics

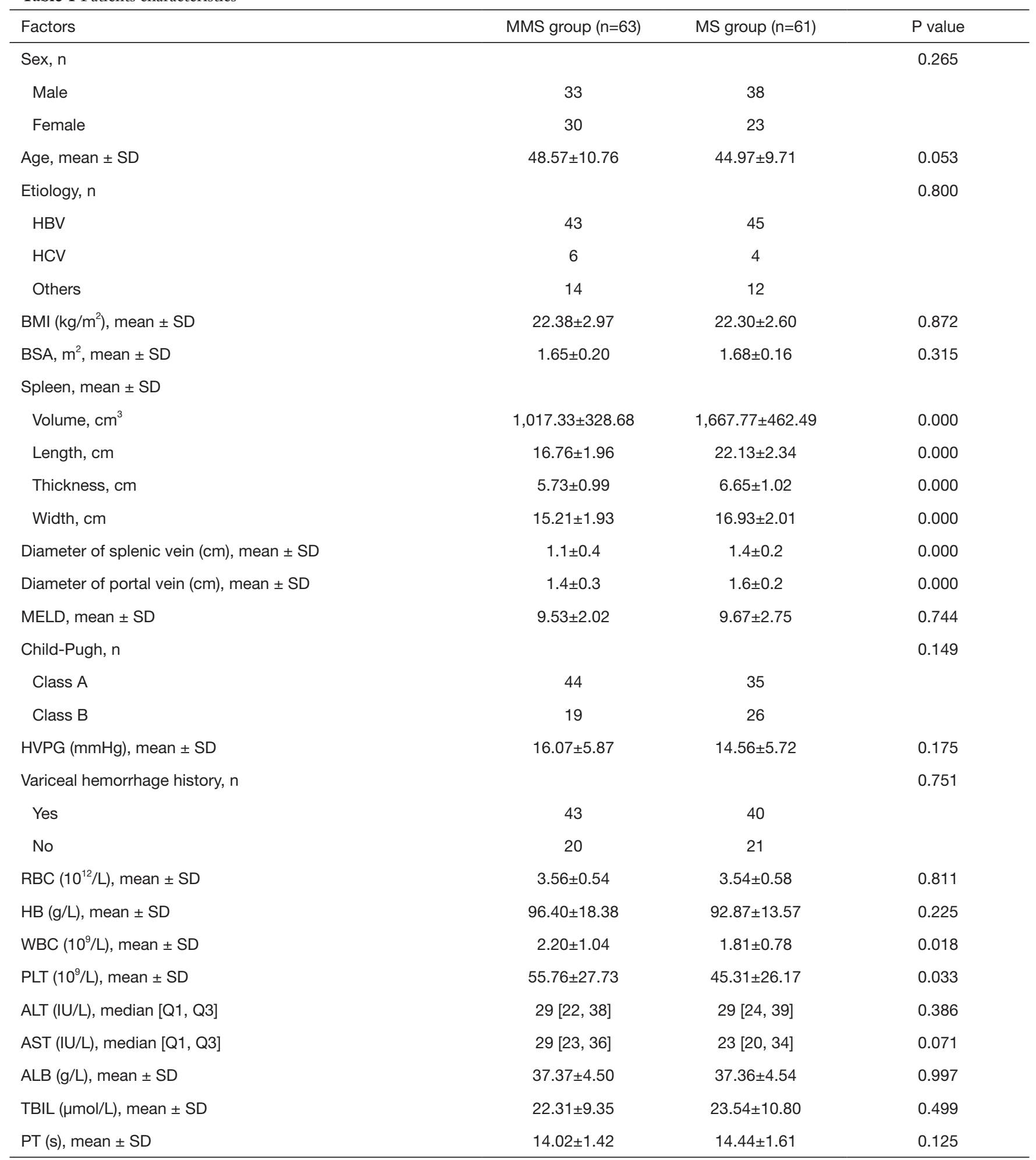

MMS, mild-to-moderate splenomegaly; MS, massive splenomegaly; HBV, hepatitis B virus; HCV, hepatitis C virus; BMI, body mass index; BSA, body surface area; MELD, Model for end-stage liver disease; HVPG, hepatic venous pressure gradient; RBC, red blood cell; HB, haemoglobin; WBC, white blood cell; PLT, platelet; ALT, alanine aminotransferase; AST, aspartate aminotransferase; ALB, albumin; TBIL, total bilirubin; PT, prothrombin time. 

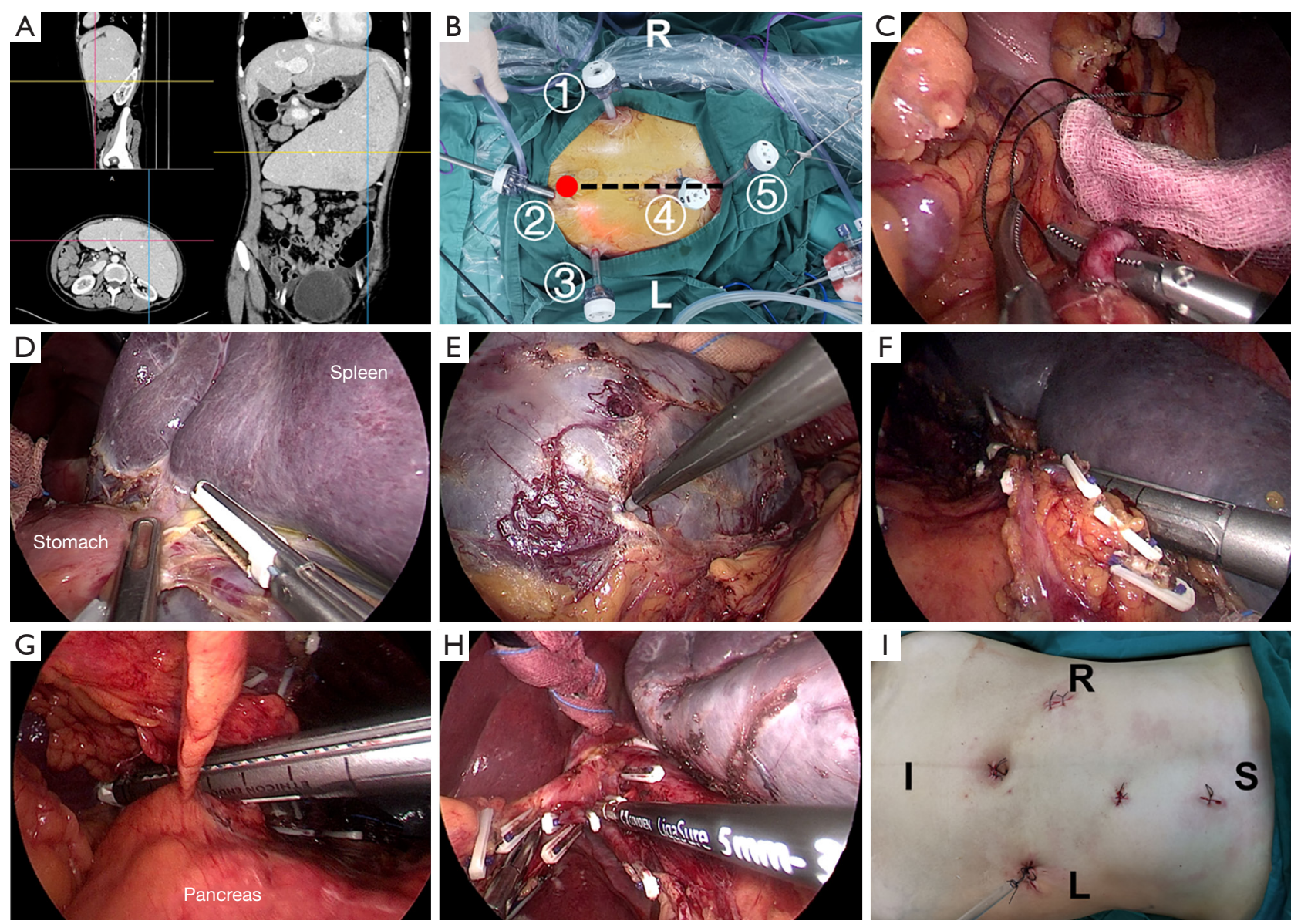

Figure 1 Evaluation and locations of the trocars and key procedures in laparoscopic splenectomy and esophagogastric devascularization. (A) Preoperative evaluation by multidetector-row computed tomography. (B) Trocar locations. 1: laparoscopic observation hole (below or to the right of the navel). 2 and 3: main operative holes. 4 and 5: secondary operative holes. The red spot shows the navel; the dotted line shows the ventral midline. (C) Separation and ligation of the splenic artery. (D) Dissection of the short gastric vessels. (E) Separation of the dorsal splenic ligament. (F) Transection of splenic hilar pedicles with the Endo GIA ${ }^{\text {TM }}$ stapler. (G) Transection of the left gastric vein at the root with the Endo GIA ${ }^{\mathrm{TM}}$ stapler. $(\mathrm{H})$ Esophagogastric devascularization. (I) Completion of totally laparoscopic surgery. R, right; L, left; S, superior; I, inferior.

supply, the spleen volume decreases, which creates more operative space and eases spleen dissection. The superior position of the spleen is deep and difficult to operate. The separation and dissection of the short gastric vessels should be performed close to the spleen in case of bleeding and gastric serosa injury (see Figure 1D). The dorsal splenic ligament was separated in the same way (see Figure 1E). The spleen was transected with an Endo GIA ${ }^{\mathrm{TM}}$ stapler $(60-2.5 \mathrm{~mm})$ after all the visible splenic ligaments had been dissected (see Figure 1F). After dissociating and cutting the left gastric vein at the root with an Endo GIA ${ }^{\mathrm{TM}}$ stapler $(45-2.5 \mathrm{~mm}$ ) (see Figure $1 G$ ), soft tissues and varicose veins were dissected along the greater and lesser curvatures of the stomach. The varices along the stomach and a segment of approximately $5 \mathrm{~cm}$ of the lower part of the esophagus were also resected (see Figure 1H). The spleen was then placed in a specimen bag in the abdominal cavity, fragmented within the specimen bag, and removed from the hole in the left lower quadrant, which was elongated to a $2-3-\mathrm{cm}$ incision. A drainage tube was placed in the left upper abdomen. The LSED procedure was thus completed (see Figure 1I).

\section{Operative outcomes and complications}

Operative outcomes, including the operation time, blood loss volume, conversion to open surgery, postoperative hospital 
stay, abdominal drainage day, and postoperative mortality rate, were recorded. Any death among the study patients that occurred in the hospital after the operation was used to calculate the mortality rate. Postoperative complications, including intra-abdominal hemorrhage, abdominal infection, pancreatic fistula, liver function impairment, severe ascites, encephalopathy, reoperation, pulmonary embolism, mortality, and gastrointestinal fistula, were also assessed. Postoperative morbidity was assessed according to the Clavien-Dindo classification (12), and $\geq$ grade III complications were defined as serious complications.

\section{Follow-up}

All the patients who underwent LSED received routine follow-up examinations. The long-term follow-up examinations were performed by telephone or at the inpatient/outpatient departments. The last follow-up examination occurred on October 10, 2021. A primary endpoint was OS, which was defined as the time from surgery to death from any cause. The other primary endpoint was recurrent hemorrhage-free survival (RHFS), which was defined as the time from surgery to first postoperative esophagogastric variceal bleeding. Patients who died of other causes or were alive at the last follow-up date were counted as censored observations.

\section{Statistical analysis}

All the analyses were performed with SPSS 25.0 software (SPSS Inc., Chicago, IL, USA). Data with a normal distribution are expressed as $\bar{x} \pm \mathrm{SD}$; those with a non-normal distribution are expressed as the median (Q1, Q3). The chi-square test was performed to compare the categorical variables. The Student's $t$ test and Mann-Whitney U test were used to compare the normally and non-normally distributed variables, respectively. Survival curves were created by KaplanMeier curves, and differences were examined by the log-rank test. A P value $<0.05$ was considered statistically significant.

\section{Results}

\section{Patient characteristics}

The baseline characteristics of the patients are summarized in Table 1. No significant differences were observed in terms of sex, age, body mass index, body surface area, etiology, history of variceal hemorrhage, liver biochemical indexes,
HVPG, and Child-Pugh score between the 2 groups. As expected, the spleen volume, length, width, and thickness of the MS group were much greater than those of the MMS group. The numbers of white blood cells and platelets were significantly lower in the MS group than the MMS group $(\mathrm{P}=0.018$ and $\mathrm{P}=0.033$, respectively). The diameters of the portal and splenic veins in the MS group were significantly larger than those of the MMS group $(\mathrm{P}=0.000)$.

\section{Perioperative outcomes}

A shorter operation time and less intraoperative blood loss were observed in 2017-2020 than 2015-2016 ( $\mathrm{P}=0.009$ and $\mathrm{P}=0.003$, respectively; see Figure 2). In all 6 years, the MS group had a longer operation time and increased intraoperative blood loss volume than the MMS group $(\mathrm{P}=0.009$ and $\mathrm{P}=0.003$, respectively). Four of the 61 patients ( 2 due to abdominal bleeding and 2 due to difficulty in separating the tissue adhesion around the spleen) in the MS group, and 0 of the 63 patients in the MMS group were converted to open surgery; however, no statistically significant difference was found between the 2 groups $(\mathrm{P}=0.119)$. Among the 4 patients converted to open surgery, 3 and 1 underwent surgery in 2015 and 2016, respectively. Despite the fact that the median time of abdominal drainage was 5 days for both groups, a significant difference was found between the 2 groups $(\mathrm{P}=0.017)$. There was no significant difference in postoperative discharge days between the 2 groups ( $\mathrm{P}=0.701$; see Table 2).

\section{Postoperative complications}

Within 30 days of surgery, 1 patient in the MS group died of abdominal infection caused by a gastric fistula. 2 patients in the MMS group (both due to abdominal bleeding) and 2 in the MS group (1 due to abdominal bleeding and 1 due to abdominal infection caused by a gastric fistula) had to undergo reoperation. The incidence rates of surgical complications (i.e., abdominal bleeding, encephalopathy, pancreatic fistula, encephalopathy, severe ascites, and liver failure) did not differ significantly between the 2 groups. Four patients in the MMS group and 8 in the MS group had serious complications; however, no significant intergroup difference was found $(\mathrm{P}=0.332$; see Table 3).

\section{Follow-up}

Fifty-four of the 63 patients $(85.7 \%)$ in the MMS group 

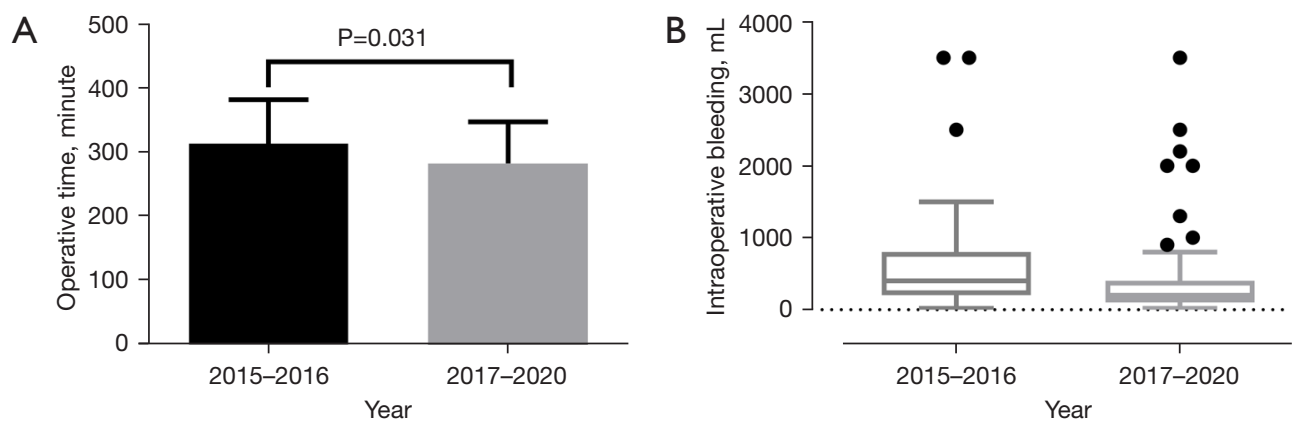

Figure 2 Comparison of operation time and intraoperative bleeding between the 2 groups in 2015-2016 and 2017-2020. (A) Operation time; (B) intraoperative bleeding.

Table 2 Perioperative data

\begin{tabular}{lccc}
\hline Perioperative data & MMS group & MS group & P value \\
\hline Operation time (min) & $275.56 \pm 57.90$ & $306.61 \pm 72.24$ & 0.009 \\
Blood loss (mL) & $200[100,400]$ & $300[200,600]$ & 0.003 \\
Abdominal drain (days) & $5[4,5]$ & $5[4,8]$ & 0.017 \\
Duration of getting out of bed (days) & $2[2,2]$ & $2[2,2]$ & 0.976 \\
Conversion to open surgery (n) & 0 & 4 & 0.119 \\
Postoperative hospital stays (days) & $8[7,9]$ & $8[7,10]$ & 0.701 \\
\hline
\end{tabular}

Data are shown as median [Q1, Q3] or mean \pm SD. MMS, mild-to-moderate splenomegaly; MS, massive splenomegaly.

had a median follow-up time of 38.2 months (range, 1.83-67.3 months). In the MS group, the follow-up rate was $91.8 \%(56 / 61)$, with a median follow-up time of 44.7 months (range, 0.33-79.5 months). Two patients died in the MS group, including 1 at postoperative day 10 (from severe abdominal infection caused by a gastric fistula), and another at postoperative day 295 (from recurrent variceal bleeding). Two patients in the MS group and 1 in the MMS group developed liver cancer and underwent surgical treatment. These 2 patients are still alive to date. During the follow-up period, 9 cases in the MMS group and 10 in the MS group showed recurrent esophagogastric variceal hemorrhage, which was controlled by drug, blood infusion, or endoscopy treatment. The 1-, 3-, and 5-year RHFS rates were $89.9 \%, 83.4 \%$, and $83.4 \%$ in the MMS group, respectively, versus $84.3 \%, 82.3 \%$, and $82.3 \%$ in the MS group, respectively. The 1-, 3-, and 5-year OS rates were all $100.0 \%$ in the MMS group, versus $98.4 \%$, $96.6 \%$, and $96.6 \%$ in the MS group, respectively. No significant differences in the follow-up data were found between the 2 groups. No patient received a liver transplantation (see
Table 4 and Figure 3).

\section{Discussion}

LS for splenectomy was first reported in 1991 (13). Since then, LS, including hand-assisted LS, has been developed as a safe procedure for the treatment of splenomegaly in liver cirrhosis patients $(14,15)$. Recently, studies have shown that LSED has obvious advantages over open surgery in portal hypertensive patients $(16,17)$. However, LS remains contradictory, especially in cases of MS with PHT $(6,18)$. This study demonstrated that the LSED procedure in MS patients with PHT is a feasible and safe treatment with satisfactory follow-up data.

LSED surgery has a sound theoretical basis and a number of advantages for the treatment of PHT. First, the splenomegaly contributes to almost $40-60 \%$ of the blood supply of the portal venous system. Thus, splenectomy reduces the portal inflow and pressure $(19,20)$. Second, targeted devascularization reduces esophagogastric variceal re-bleeding. Third, the normalization of the shear stress of the portal pressure helps 
Table 3 Postoperative complications

\begin{tabular}{|c|c|c|c|}
\hline Postoperative complications & MMS group $(n=63)$ & MS group $(n=61)$ & $P$ value \\
\hline Intra-abdominal infection, n (\%) & 0 & $2(3.3)$ & 0.240 \\
\hline 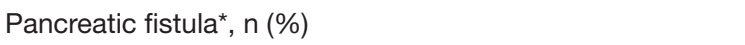 & 0 & $1(1.6)$ & 0.492 \\
\hline Postoperative liver failure, $\mathrm{n}(\%)$ & 0 & 0 & 1 \\
\hline Encephalopathy, n (\%) & 0 & $1(1.6)$ & 0.492 \\
\hline Reoperation after LSED, n (\%) & $2(3.2)$ & $2(3.3)$ & 0.634 \\
\hline Pulmonary embolism, n (\%) & $1(1.6)$ & 0 & 1.000 \\
\hline Mortality, n (\%) & 0 & $1(1.6)$ & 0.492 \\
\hline IIIB & 2 & 2 & 0.634 \\
\hline IVA & 2 & 2 & 0.634 \\
\hline IVB & 0 & 1 & 0.492 \\
\hline $\mathrm{V}$ & 0 & 1 & 0.492 \\
\hline
\end{tabular}

*, according to the International Study Group of Pancreatic fistula. MMS, mild-to-moderate splenomegaly; MS, massive splenomegaly; LSED, laparoscopic splenectomy and esophagogastric devascularization.

improve liver function, and splenectomy eliminates a large amount of inflammatory factors that worsen liver function and cause liver fibrosis $(21,22)$. Fourth, splenectomy decreases the risk of hepatocellular carcinoma in patients with post-hepatitis cirrhosis (23).

Spleen size is a major factor limiting the laparoscopic operation, but should not be considered the only determinant to decide whether the laparoscopic surgery is performed. In this study, all 4 patients who were converted to open surgery were in the MS group, which does show that a massive spleen causes some trouble in laparoscopic operations. In 2 of the cases, the issues were related to the insufficient space and the difficulty of the operation; however, in the other 2 cases, the issue was related to severe adhesion around the spleen. Based on our experience, it would be unscientific to rely solely on spleen size as the standard for determining the feasibility of laparoscopic surgery. The degree of adhesion around the spleen tissue is also a major factor affecting laparoscopic surgery. Many patients have fibrous exudation on the spleen surface, resulting in close adhesion between the spleen and surrounding tissues. Additionally, some patients have a history of endoscopic hypertonic glucose tissue adhesion, sclerotherapy injection or splenic artery embolization before operation, which hardens the perigastric tissue and induces inflammatory reactions. This would obviously increase the operation difficulty and bleeding.

Intraoperative bleeding and operation time were more increased in the MS group than the MMS group, but they were still within the scope of operation safety and operability. Intraoperative abdominal bleeding is a major cause of conversion to open surgery. It has been reported that a spleen length $\geq 19 \mathrm{~cm}$ is a risk factor for difficult LS and intraoperative hemorrhage (24). No significant difference in HVPG was found between the 2 groups; however, the diameters of the splenic and portal veins in the MS group were much larger than those of the MMS group, indicating that more portal-systemic collaterals formed in the MS group. Both MS and the enhanced formation of collateral veins create greater challenges in laparoscopic surgery. Splenic artery ligation is the key and primary step for ensuring the safety of the LS operation. Without blood supply to the spleen, the spleen softens and reduces in volume, which makes the operation easier to perform, reduces the operative bleeding, and ensures the safety of the operation. The short gastric veins and collateral vessels in PHT patients are often 
Table 4 Follow-up data

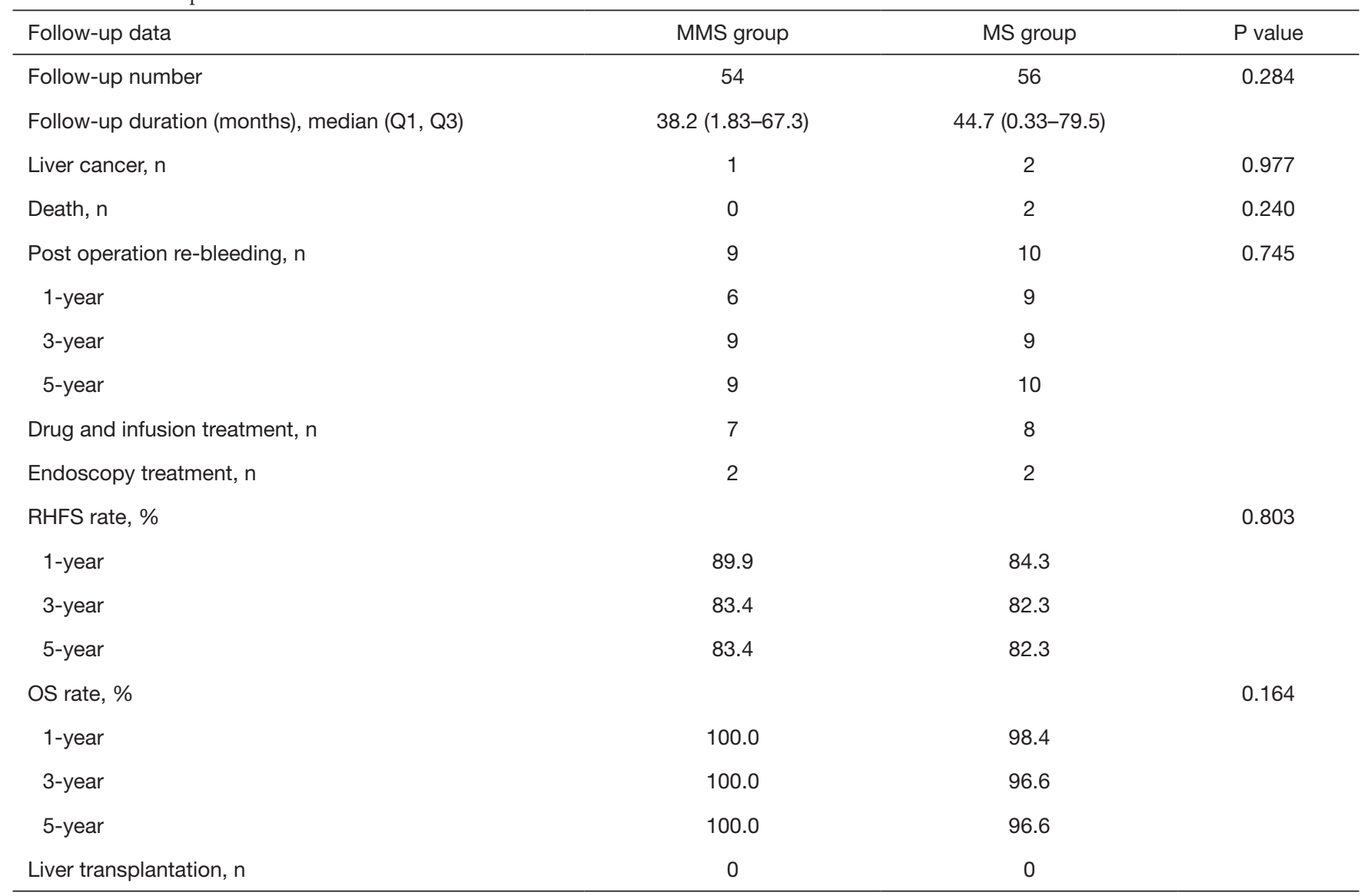

MMS, mild-to-moderate splenomegaly; MS, massive splenomegaly; RHFS, recurrent hemorrhage-free survival; OS, overall survival.
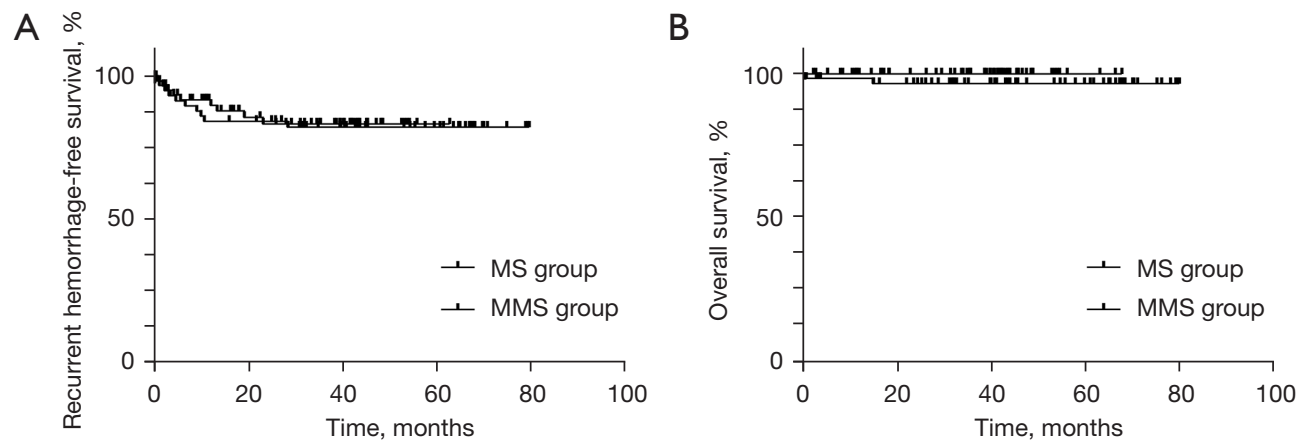

Figure 3 The follow-up data between the 2 groups. (A) Recurrent hemorrhage-free survival; (B) overall survival. MS, massive splenomegaly; MMS, mild-to-moderate splenomegaly.

abnormally dilated. Additionally, due to the deep position and narrow operative space, the superior position of the spleen is prone to bleeding during surgery. Thus, the operation should be close to the spleen to dissect the gastrosplenic ligament and to ligate the short gastric vein. It is necessary to change the position of the trocars appropriately according to spleen size and position, especially in MS treatment. A trocar can first be inserted for the observation hole, and other trocars can then be arranged according to spleen position and size. The proper dissociation of the spleen-stomach ligament 
and the lower spleen ligament could increase the activity of the spleen. The assistant can press the spleen on the lateral abdomen to increase the abdominal space for the main surgeon to ligate the splenic artery.

Good surgical team experience and cooperation are particularly important for any operation, especially in endoscopic surgery (25). In this study, the intraoperative bleeding and operation time were significantly lower in 2017-2020 than 2015-2016. Further, all 4 cases of conversion to open surgery occurred in 2015 and 2016. These findings indicate significant improvements in the cooperation of the surgical team and their surgical experience, especially in relation to LS for MS.

Postoperative intra-abdominal bleeding is an important factor contributing to reoperation. In this study, 2 of the 63 patients in the MMS group and 7 of the 61 patients in the MS group had intra-abdominal bleeding postoperatively. However, 4 patients required reoperation for hemorrhage control. Surgical wound bleeding is an important cause of abdominal bleeding. Due to dysregulated coagulation and high portal vein pressure, patients with PHT are more prone to postoperative abdominal bleeding than other patients. Thus, we preferred the LigaSure vessel-sealing system to the harmonic scalpel to occlude the ligaments and small vessels. Another unnoticed cause leading to abdominal bleeding is trocar-site bleeding. Due to the dilation of abdominal wall veins and dysregulated coagulation function in patients with PHT, it is difficult to stop bleeding, even bleeding from a small vascular injury, in the trocar site, which may ultimately lead to intra-abdominal bleeding and reoperation. In this study, 2 of the 4 patients who required surgical hemostasis had confirmed abdominal bleeding caused by trocar-site bleeding. Thus, it is very important to completely suture the peritoneum of the trocar site to restore its integrity.

Infection is a risk factor for postoperative morbidity and mortality (26). Intra-abdominal infection, caused by bacterial translocation or products from the intestinal lumen, is more prominent in decompensated cirrhotic PHT patients compared to patients without PHT (27). Theoretically, patients with liver cirrhosis undergoing abdominal surgery have a higher incidence of abdominal infection than normal patients. In this study, postoperative intra-abdominal infection occurred in 2 patients in the MS group, 1 of whom died of a gastric fistula. Based on the video of the operation, the serous membrane on the greater curvature of the stomach may have been damaged when dissecting the short gastric vein on the upper pole of the spleen. Additionally, the patient did not follow the doctor's advice and ate a great deal on the $2^{\text {nd }}$ day postoperatively, which resulted in rapid gastric expansion, and ultimately a gastric fistula. Thus, due to the narrow operation space of the upper pole of the spleen, the division should be close to the spleen; in case of possible gastric serosa injury, it should be sutured during the operation.

Postoperative esophagogastric variceal re-bleeding and mortality are important indexes for evaluating the curative effect of surgery. Endoscopic treatment is the first line of treatment for variceal bleeding, but still has a cumulative re-bleeding rate of $25-30 \%$, and continuous endoscopic treatments are needed (28). Transjugular intrahepatic portosystemic shunt (TIPS) is also effective at reducing the incidence of cirrhosis variceal re-bleeding, but still has a rebleeding rate of $20-30 \%$ (29). The postoperative analysis in this study showed a low cumulative esophagogastric variceal hemorrhage rate, but 19 patients still experienced rebleeding. This may be due to the LSED operation cutting off the variceal veins outside the gastroesophageal tract, and the poor disconnection of the variceal veins coming from the gastric submucosa. Continuous endoscopic treatment after LSED may help to further decrease the rebleeding rate, but it requires further exploration. TIPS was shown to be more effective in reducing the incidence of cirrhotic esophagogastric variceal re-bleeding, and re-bleeding-related mortality, and overall mortality in cirrhosis (30). Early TIPS offers survival benefits for Child-Pugh C cirrhosis cases, but not Child-Pugh A or B cases (31). In this study, patients with Child-Pugh A and B who underwent LSED had a satisfactory OS rate. This may be because LSED not only decreases variceal re-bleeding but also contributes to the improvement of liver function and produces beneficial immunological changes $(32,33)$. However, the question of whether the LSED operation is conducive to long-term survival in decompensated cirrhotic patients with Child-Pugh A and B requires further exploration.

In conclusion, the LSED procedure was found to be associated with increased intraoperative blood loss and a longer operation time in the MS group than the MMS group, but it is still technically feasible and safe for the treatment of MS patients with PHT. MS does not increase the rate of conversion to open surgery and perioperative complications under an experienced surgical team with fine perioperative planning and management. The LSED operation has a low re-bleeding rate and a satisfactory survival time in patients with PHT. 


\section{Acknowledgments}

Funding: The study was supported by the National Natural Science Foundation of China (No. 81700533); the Key Research and Development Program of Shaanxi Province, China (Nos. 2017SF-116, 2015JM8420, 2015KTCL03-05); the Innovation and Development Foundation of Tangdu Hospital (Nos. 2017LCYJ003, 2018JSYJ010, 2019LCYJ005).

\section{Footnote}

Reporting Checklist: The authors have completed the STROBE reporting checklist. Available at https://atm. amegroups.com/article/view/10.21037/atm-22-502/rc

Data Sharing Statement: Available at https://atm.amegroups. com/article/view/10.21037/atm-22-502/dss

Conflicts of Interest: All authors have completed the ICMJE uniform disclosure form (available at https://atm. amegroups.com/article/view/10.21037/atm-22-502/coif). The authors have no conflicts of interest to declare.

Ethical Statement: The authors are accountable for all aspects of the work in ensuring that questions related to the accuracy or integrity of any part of the work are appropriately investigated and resolved. All procedures performed in this study involving human participants were in accordance with the Declaration of Helsinki (as revised in 2013). This study was approved by the Ethical Committee of Tangdu Hospital (approval No. 202011-32) and informed consent was taken from all the patients.

Open Access Statement: This is an Open Access article distributed in accordance with the Creative Commons Attribution-NonCommercial-NoDerivs 4.0 International License (CC BY-NC-ND 4.0), which permits the noncommercial replication and distribution of the article with the strict proviso that no changes or edits are made and the original work is properly cited (including links to both the formal publication through the relevant DOI and the license). See: https://creativecommons.org/licenses/by-nc-nd/4.0/.

\section{References}

1. Yang Z, Qiu F. Pericardial Devascularization with Splenectomy for the Treatment of Portal Hypertension.
Chinese Journal of Surgery 2000;38:645-8.

2. Yang L, Zhang Z, Zheng J, et al. Long-term outcomes of oesophagogastric devascularization and splenectomy in patients with portal hypertension and liver cirrhosis. ANZ J Surg 2020;90:2269-73.

3. Zhao Y, Wang C. The therapeutic effect of splenectomy plus selective pericardial devascularization versus conventional pericardial devascularization on portal hypertension in China: a meta-analysis. Oncotarget 2018;9:15398-408.

4. Luo HP, Zhang ZG, Long X, et al. Combined Laparoscopic Splenectomy and Esophagogastric Devascularization versus Open Splenectomy and Esophagogastric Devascularization for Portal Hypertension due to Liver Cirrhosis. Curr Med Sci 2020;40:117-22.

5. Lin J, Liu Q, Liang Z, et al. Laparoscopic selective esophagogastric devascularization and splenectomy for patients with cirrhotic portal hypertension. Wideochir Inne Tech Maloinwazyjne 2019;14:187-94.

6. Deng ZC, Jiang WZ, Chen L, et al. Laparoscopic VS. Open splenectomy and oesophagogastric devascularisation for liver cirrhosis and portal hypertension: A retrospective cohort study. Int J Surg 2020;80:79-83.

7. Habermalz B, Sauerland S, Decker G, et al. Laparoscopic splenectomy: the clinical practice guidelines of the European Association for Endoscopic Surgery (EAES). Surg Endosc 2008;22:821-48.

8. Tsamalaidze L, Stauffer JA, Permenter SL, et al. Laparoscopic Splenectomy for Massive Splenomegaly: Does Size Matter? J Laparoendosc Adv Surg Tech A 2017;27:1009-14.

9. Casaccia M, Sormani MP, Palombo D, et al. Laparoscopic Splenectomy Versus Open Splenectomy In Massive and Giant Spleens: Should we Update the 2008 EAES Guidelines? Surg Laparosc Endosc Percutan Tech 2019;29:178-81.

10. Tripathi D, Stanley AJ, Hayes PC, et al. U.K. guidelines on the management of variceal haemorrhage in cirrhotic patients. Gut 2015;64:1680-704.

11. Augustin S, Pons M, Maurice JB, et al. Expanding the Baveno VI criteria for the screening of varices in patients with compensated advanced chronic liver disease. Hepatology 2017;66:1980-8.

12. Clavien PA, Barkun J, de Oliveira ML, et al. The ClavienDindo classification of surgical complications: five-year experience. Ann Surg 2009;250:187-96.

13. Delaitre B, Maignien B. Splenectomy by the laparoscopic 
approach. Report of a case. Presse Med 1991;20:2263.

14. Guadagni S, Gianardi D, Morelli L. Hand-Assisted Splenic Bed Laparoscopic Splenectomy for Massive Splenomegaly Secondary to Portal Hypertension and Liver Cirrhosis. Am Surg 2019;85:e271-2.

15. Kawanaka H, Akahoshi T, Kinjo N, et al. Laparoscopic Splenectomy with Technical Standardization and Selection Criteria for Standard or Hand-Assisted Approach in 390 Patients with Liver Cirrhosis and Portal Hypertension. J Am Coll Surg 2015;221:354-66.

16. Zheng S, Sun P, Liu X, et al. Efficacy and safety of laparoscopic splenectomy and esophagogastric devascularization for portal hypertension: A single-center experience. Medicine (Baltimore) 2018;97:e13703.

17. Chen H, Yang F, Li TT, et al. Comparison of Efficacy of Laparoscopic and Open Splenectomy Combined With Selective and Nonselective Pericardial Devascularization in Portal Hypertension Patients. Surg Laparosc Endosc Percutan Tech 2018;28:401-3.

18. Shin RD, Lis R, Levergood NR, et al. Laparoscopic versus open splenectomy for splenomegaly: the verdict is unclear. Surg Endosc 2019;33:1298-303.

19. Kawanaka H, Akahoshi T, Kinjo N, et al. Effect of laparoscopic splenectomy on portal haemodynamics in patients with liver cirrhosis and portal hypertension. Br J Surg 2014;101:1585-93.

20. Ni YB, Gao PJ, Wang D, et al. Esophagogastric devascularization without splenectomy in portal hypertension: safe and effective? Hepatobiliary Pancreat Dis Int 2015;14:276-80.

21. Yamamoto N, Okano K, Oshima M, et al. Laparoscopic splenectomy for patients with liver cirrhosis: Improvement of liver function in patients with Child-Pugh class B. Surgery 2015;158:1538-44.

22. Huang N, Ji F, Zhang S, et al. Effect of Splenectomy on Serum Cytokine Profiles in Hepatitis B Virus-Related Cirrhosis Patients with Portal Hypertension. Viral Immunol 2018;31:371-8.

23. Lv X, Yang F, Guo X, et al. Hypersplenism is correlated with increased risk of hepatocellular carcinoma in patients with post-hepatitis cirrhosis. Tumour Biol 2016;37:8889-900.

24. Wysocki M, Radkowiak D, Zychowicz A, et al. Prediction of Technical Difficulties in Laparoscopic Splenectomy and Analysis of Risk Factors for Postoperative Complications in 468 Cases. J Clin Med 2018;7:547.

25. Wu Z, Zhou J, Cai YQ, et al. The learning curve for laparoscopic splenectomy for massive splenomegaly: a single surgeon's experience. Chin Med J (Engl) 2013;126:2103-8.

26. De Simone B, Sartelli M, Coccolini F, et al. Intraoperative surgical site infection control and prevention: a position paper and future addendum to WSES intra-abdominal infections guidelines. World J Emerg Surg 2020;15:10.

27. Turco L, Garcia-Tsao G. Portal Hypertension: Pathogenesis and Diagnosis. Clin Liver Dis 2019;23:573-87.

28. Al-Khazraji A, Curry MP. The current knowledge about the therapeutic use of endoscopic sclerotherapy and endoscopic tissue adhesives in variceal bleeding. Expert Rev Gastroenterol Hepatol 2019;13:893-7.

29. Hosokawa I, Adam R, Allard MA, et al. Outcomes of surgical shunts and transjugular intrahepatic portasystemic stent shunts for complicated portal hypertension. Br J Surg 2017;104:443-51.

30. Jing L, Zhang Q, Chang Z, et al. Nonsurgical Secondary Prophylaxis of Esophageal Variceal Bleeding in Cirrhotic Patients: A Systematic Review and Network Meta-analysis. J Clin Gastroenterol 202 1;55:159-68.

31. Lv Y, Zuo L, Zhu X, et al. Identifying optimal candidates for early TIPS among patients with cirrhosis and acute variceal bleeding: a multicentre observational study. Gut 2019;68:1297-310.

32. Huang N, Ji FP, Zhang S, et al. Spleen-Associated Effects on Immunity in Hepatitis B Virus-Related Cirrhosis with Portal Hypertension. J Interferon Cytokine Res 2019;39:95-105.

33. Nomura Y, Kage M, Ogata T, et al. Influence of splenectomy in patients with liver cirrhosis and hypersplenism. Hepatol Res 2014;44:E100-9.

(English Language Editor: L. Huleatt)

Cite this article as: Wang D, Chen $X, L v$, Yang T, Huang B, Cao Y, Lu J, Yin J. Laparoscopic splenectomy and devascularization for massive splenomegaly in portal hypertensive patients: a retrospective study of a single surgical team's experience with 6-year follow-up data. Ann Transl Med 2022;10(4):207. doi: 10.21037/atm-22-502 